\title{
Lidil
}

Revue de linguistique et de didactique des langues

$60 \mid 2019$

Langues des signes et genres discursifs

\section{Aborder la sensibilisation aux variétés du français en A2-B1 : enjeux et opportunités}

Integrating Varieties of French at A2-B1 Level: Challenges and Opportunities

\section{Christèle Maizonniaux}

\section{(2) OpenEdition}

1 Journals

Édition électronique

URL : http://journals.openedition.org/lidil/6598

DOI : 10.4000/lidil.6598

ISSN : 1960-6052

Éditeur

UGA Éditions/Université Grenoble Alpes

Édition imprimée

ISBN : 978-2-37747-164-5

ISSN : $1146-6480$

Référence électronique

Christèle Maizonniaux, «Aborder la sensibilisation aux variétés du français en A2-B1 : enjeux et opportunités », Lidil [En ligne], 60 | 2019, mis en ligne le 01 novembre 2019, consulté le 13 avril 2020. URL : http://journals.openedition.org/lidil/6598; DOI : https://doi.org/10.4000/lidil.6598

Ce document a été généré automatiquement le 13 avril 2020

(C) Lidil 


\title{
Aborder la sensibilisation aux variétés du français en A2-B1 : enjeux et opportunités
}

\author{
Integrating Varieties of French at A2-B1 Level: Challenges and Opportunities
}

\author{
Christèle Maizonniaux
}

\section{Introduction}

1 La question de l'enseignement de la variation en français langue étrangère est une question d'actualité. En effet, plusieurs ouvrages ou articles récemment parus (Detey et coll., 2010 ; Detey \& Racine, 2012 ; Galazzi \& Paternostro, 2016; Falkert, 2016a ; Galazzi, 2017 ; Tyne et coll., 2017 ; Jeanmaire, 2017) abordent la question et soulèvent divers axes de réflexion sociolinguistiques ou didactiques. On remarque également l'organisation de plusieurs manifestations scientifiques telles que le colloque «Prononcer les langues : variations, émotions, médiations » (Rouen, octobre 2017) ou encore le colloque «Métissages linguistiques et culturels émergents: vers quelles médiations?» (Paris-Est Créteil, octobre 2017). Les arguments en faveur de l'élargissement de l'enseignement du français aux variations présentes dans le monde francophone sont divers. Cela peut contribuer à l'attractivité de la langue française (Fréchet, 2016, p. 14), à refléter de manière plus juste «la réalité du français tel qu'il existe » (Sheeren, 2012), ou encore à faciliter l'apprentissage du FLE (Jeanmaire, 2017).

2 Adopter au niveau A2-B1 une vision élargie de l'enseignement du français, s'intéresser à ses variations, qu'elles soient phonétiques, lexicales, syntaxiques, morphologiques voire pragmatiques renvoie à la question des normes pédagogiques pour l'enseignement de l'oral (Valdman, 1989). Plusieurs questions se posent : traitera-t-on, à ce niveau, d'un aspect de la variation plutôt que d'un autre? Si l'on décide d'en retenir plusieurs, dans quel ordre est-il préférable de les traiter ? Et, faut-il à ce niveau se cantonner à la compréhension plutôt qu'à la production? 
3 Mon propos est ici de présenter trois propositions didactiques mises en œuvre en contexte universitaire australien auprès d'apprenants de niveau A2-B1 tout en questionnant leur pertinence par rapport aux questions qui traversent actuellement le champ.

\section{Arrière-plan théorique}

\subsection{Pourquoi s'intéresser à la variation en A2-B1 ?}

Ces dernières années, un certain nombre de chercheurs dans le champ du français langue étrangère mettent en évidence la nécessité d'une prise en compte des variétés du français allant au-delà d'une simple présentation de faits culturels, et ce, à des niveaux peu élevés de maitrise de la langue cible. Ainsi Falkert (2016a) et Sheeren (2012) prônent une sensibilisation à la variété diatopique du français - autrement dit une sensibilisation aux accents régionaux et aux accents des pays francophones - très tôt dans l'apprentissage. Constatant que le cadre européen ne propose une sensibilisation à la variation qu'en B2, Falkert (2016b) affirme: «[...] l'apprenant sera confronté à la variation au moment même où il devra interagir à l'oral avec un locuteur natif, qu'il ait atteint le niveau B2 ou non.»(p.159) Parlant du français standard, elle ajoute "c'est son statut de point de repère unique jusqu'au niveau B2 qui pose problème » (p. 163).

5 Auzeau (2016) et d'autres font le même constat. Il est évident que les apprenants de français sur objectifs spécifiques (FOS) d'Auzeau (des religieux sur le point de partir en mission en Afrique francophone) ont besoin d'être sensibilisés à la variation, et ce, alors qu'ils n'ont atteint que le niveau B1. Dans le cas d'Auzeau, comme dans d'autres, les apprenants ne poursuivent pas nécessairement leur apprentissage formel du français au-delà de B2, et n'auront donc pas eu l'occasion d'être sensibilisés aux accents francophones.

6 Les travaux de l'équipe I-PFC (Inter-Phonologie du français contemporain) sont intéressants pour nous car ils offrent une base de données de français oral dans l'espace francophone. Il faut en outre noter que cette équipe propose des fiches pédagogiques pour les enseignants illustrant diverses variétés de français ${ }^{1}$.

7 Valdman (2000), qui envisage une sensibilisation précoce à la variété diatopique (lexique et particularités phonologiques) indique qu'il «ne s'agit pas de faire acquérir ces variantes aux apprenants mais de les leur faire lire et entendre tout en, si possible, les signalant et les commentant » (p. 650). Valdman note également la nécessité pour l'enseignant d'avoir une certaine connaissance des diverses situations du français en francophonie et la capacité d'identifier certains traits diatopiques marquants. Il note en outre que deux écueils doivent être évités dans la présentation de la variété diatopique : la caricature ou la stéréotypie ainsi que l'application réductionniste de la norme au seul français standard.

\subsection{La variation dans les outils de référence et les manuels}

Le CECR ne préconise rien qui aille au-delà d'une sensibilisation aux spécificités culturelles en A2-B1. C'est seulement lorsque l'apprenant a atteint le niveau B2 qu'est prévue une sensibilisation à la variation langagière ${ }^{2}$. Ainsi que le note Falkert (2016b), 
« le passage du niveau B2 au niveau $\mathrm{C} 1$ constitue une étape cruciale dans la mesure où l'apprenant sera amené à identifier et à décoder des énoncés en langue non standard " (p. 165).

Il faut cependant remarquer que le Cadre de référence pour les approches plurielles (CARAP), publié par le Centre européen pour les langues vivantes, propose des activités didactiques basées sur plusieurs variétés de langues et de cultures et notamment des activités interculturelles invitant les apprenants à travailler sur des textes d'Afrique francophone tels que des contes sénégalais.

Le nouveau curriculum australien pour le primaire et le secondaire (Australian Curriculum, Assessment and Reporting Authority [ACARA], 2014) intègre une sensibilisation à la variation dès le début de l'apprentissage ${ }^{3}$. L'objectif fixé dans la rubrique compréhension est de comprendre comment la langue varie dans son utilisation (registre, style, variétés standard et non standard) et en fonction du lieu et de l'époque. Pour Angela Scarino, responsable de l'équipe de rédaction de ces programmes, il convient de s'écarter d'un enseignement des langues basé sur les règles tel qu'il régnait par le passé, pour s'intéresser à la variation ${ }^{4}$. Il est intéressant de noter que la souscatégorie "language variation and change» est intégrée dans une perspective interculturelle plus large ${ }^{5}$.

11 Pour Grimoldi (2014), les manuels de FLE se concentrent surtout sur les aspects culturels de pays choisis (essentiellement le Québec et la Suisse), la variation n'y est abordée que de manière anecdotique, et dans tous les ouvrages examinés, majoritairement en relation avec le lexique. Pour Sheeren (2016a), « les dialogues, les situations proposées, les activités didactiques sont empreintes d'un lutéciotropisme excessif» (p. 75). Sheeren souligne également le fait que le métissage actuel de la France - et de sa capitale - est passé sous silence et que les ouvrages ne prennent pas en considération la variété des cultures présentes sur le territoire (voir aussi Paternostro (2016) et Gadet (2017)). En outre, ce même chercheur note que le lexique des manuels de FLE « est exclusivement centré sur celui de l'Hexagone et il est donné pour acquis qu'il est compris et utilisé par toute la francophonie » (Sheeren, 2016a, p. 74). Certains termes proposés dans les manuels tels que le vocabulaire de l'école ou le verlan « doivent être considérés comme des francismes, quoique les manuels tendent à les faire passer pour des termes standards, universels, dénués de toute connotation géographique $^{6} »($ ibid.).

On peut cependant remarquer que plusieurs manuels ou matériaux pédagogiques récents intègrent la variation. On citera à titre d'exemple, Clic-Ado (<www.elionline.com/eng/book-details?2745/free>), qui présente quatre personnages de diverses régions de la francophonie du Nord : une Française d'Aix-en-Provence, une Suisse, un Belge et un Québécois ou encore les documents produits par l'université des langues étrangères de Tokyo (TUFS), plutôt destinés aux niveaux intermédiaires et débutants ${ }^{7}$.

\subsection{Exemples d'activités proposées dans différents manuels}

Baser une démarche pédagogique sur la littérature patrimoniale peut permettre de mettre en place une sensibilisation aux accents et/ou au lexique d'une région francophone. Trottet et Amireault (2015) et Amireault et coll. (n. d.) proposent d'entrer dans l'étude du conte québécois La Chasse-galerie, par le visionnement d'un ou de deux 
films d'animation ${ }^{8}$ présentant une variété québécoise, ou par l'écoute d'une chanson québécoise. Le travail proposé prend également en compte le lexique spécifique du conte.

L'ouvrage d'Isabelle Maingain, Le français d'ici et d'ailleurs (2006), propose des exercices de grammaire basés sur des textes produits dans différentes régions francophones et met en relation, ainsi que le remarque Sheeren (2012), des anglicismes utilisés en France et leurs équivalents québécois.

De Pietro, Diemoz et Matthey (2016) proposent divers exercices qui invitent à mettre en relation des mots et expressions de Suisse romande et leurs équivalents en français standard. Ils suggèrent, en contexte suisse francophone, de reprendre le «mécanisme du détour ", afin de "faire un détour par d'autres (variétés de) langues pour mieux revenir à une réflexion portant sur sa propre (variété de) langue » (Perregaux et coll., 2003 ; De Pietro, 2004).

Auzeau (2016) propose divers exercices faisant appel à la dimension corporelle. Ceux-ci sont précédés d'activités visant à assurer la compréhension lexicale (à travers l'écoute et la transcription d'enregistrements), à effectuer une observation prosodique et phonologique de type comparatif avant de passer à une phase de réexploitation et d'imitation des modèles sonores entendus. Cette dernière phase, inspirée des travaux de Pillot-Loiseau (2014), invite les apprenants placés en cercle à marquer le rythme des phrases d'un mouvement de main puis à travailler en binôme avec un système de tapemain.

Jeanmaire (2017), qui considère les variétés du français comme « un outil pédagogique aux multiples vertus » (p. 158), invite ses apprenants débutants à former des groupes de travail et à présenter à l'oral une célébrité francophone. Dans cette approche, il a recours à de multiples ressources en lignes (webdocumentaires de TV5 monde, extraits vidéo, etc.). Pour ses apprenants faux-débutants, il envisage un jeu de rôle avec guide et touristes autour de monuments célèbres de pays francophones. Au niveau intermédiaire, il propose à ses apprenants d'interviewer et de filmer un francophone, de s'investir dans un projet de collaboration avec un francophone, ou dans la réalisation d'une vidéo présentant une recette de cuisine d'un espace francophone. Au niveau avancé enfin, il procède à un travail plus systématique à partir de ressources conçues spécifiquement et d'extraits de films. Pour réaliser ces tâches, chaque groupe d'apprenants, quel que soit son niveau, travaille avec des ressources authentiques et se trouve donc exposé à la variation, notamment lexicale et phonétique/phonologique.

\section{Trois exemples de mise en œuvre au niveau A2-B1}

\subsection{Une approche spécifique visant la variation}

Cette approche a été élaborée pour répondre aux objectifs suivants : sensibiliser à la diversité de la langue française et à son caractère dynamique, interroger la notion de français standard, montrer que les spécificités lexicales et phonologiques de la francophonie participent de la portée culturelle des œuvres ou documents étudiés. Il s'agit également, dans le cadre de cette sensibilisation, de mettre à profit les apports de l'image pour la compréhension du lexique et des accents puisque les divers supports proposés sont multimodaux (albums pour la jeunesse d'une part, vidéo d'autre part). 
19 S'il est légitime de s'interroger sur la nature et la forme que peut prendre une progression dans le cadre d'une sensibilisation aux accents, à la morphosyntaxe ou au lexique, il nous faut préciser que les expérimentations présentées ici ne se sont pas inscrites dans une progression. Elles ont été envisagées de manière séparée, même si les travaux réalisés à partir de supports pour la jeunesse font partie d'un seul et même module ayant pour objectif une ouverture à la francophonie.

Nous avons fait le choix de nous focaliser sur la réception et non sur la production. Celle-ci pourrait en effet être perçue comme une complexification inutile par des apprenants de niveau A2-B1. L'objectif des activités est de les sensibiliser à une dimension peu connue du français.

21 Les activités proposées touchent au lexique et aux accents. Elles se basent d'une part sur des albums pour la jeunesse suisse et québécois et d'autre part sur un court métrage québécois, tiré de l'album pour la jeunesse étudié préalablement en classe. Par ailleurs, dans un autre contexte universitaire, mais toujours auprès d'apprenants de niveau A2B1, nous avons proposé une sensibilisation à l'accent camerounais par le biais d'une vidéo ${ }^{\text {. }}$

\subsection{Questions préalables}

22 Les questions suivantes sous-tendent notre réflexion:

- Quels sont les supports et les démarches appropriés pour une sensibilisation à la variété lexicale et aux accents régionaux de la francophonie à ce niveau de maitrise de la langue cible?

- En quoi les supports pour la jeunesse peuvent-ils constituer des supports adaptés, et ce, alors qu'à priori, ils ne sont pas destinés à un public adulte? Autrement dit, dans quelle mesure des éléments spécifiques à ces supports (tels que glossaire, images ou adaptations filmiques) peuvent-ils constituer des ressources didactiques pour une approche en A2-B1?

- En quoi de telles démarches peuvent-elles, à ce niveau, compléter l'enseignement des autres domaines de la variation (registres de langues, distinction entre oral et écrit notamment) ?

\subsection{Sensibilisation à des accents régionaux de la francophonie (Québec et Cameroun)}

23 Les deux propositions pédagogiques décrites ci-dessous sont basées sur des documents vidéo disponibles sur le web. L'objectif est de sensibiliser les apprenants de niveau A2B1 à des accents du Québec et du Cameroun.

\subsubsection{Un court métrage québécois basé sur un album pour la jeunesse}

24 La sensibilisation à la variété québécoise se fait à partir du visionnement du cours métrage réalisé par Sheldon Cohen (1980), adaptation d'un classique de la littérature de jeunesse, l'album Le chandail de hockey de Roch Carrier et Sheldon Cohen (1985, première édition : 1979). Le court métrage présente plusieurs éléments facilitateurs pour les apprenants ayant préalablement étudié l'album éponyme. Ils retrouvent les images vues et découvrent une bande son présentant, outre le français parlé avec un accent québécois, un certain nombre de bruitages dont la présence permet d'inférer le sens du texte entendu et de mieux situer celui-ci dans la trame narrative. Visionner le 
court métrage accroit la portée culturelle de l'album étudié ${ }^{10}$. Il ne s'agit plus simplement de voir et d'imaginer ce qui est décrit dans l'album. Les apprenants sont à proprement parler, plongés dans l'ambiance, les sons et les intonations québécoises. La phase préparatoire au cours de laquelle ceux-ci ont pris connaissance de l'album, fait quelques recherches sur les personnages présentés et échangé sur l'ouvrage et le contexte historique représenté, nous parait cruciale. Sans cette phase préparatoire, il est probable que la compréhension du document vidéo aurait été difficile, tant au niveau lexical qu'au niveau de la prononciation ou de la compréhension de son contenu culturel $^{11}$.

L'exposition à l'accent québécois apparait donc en phase finale de la démarche et reste une sensibilisation: aucun travail de repérage des traits phonétiques/phonologiques n'a été mené.

Les réactions positives des apprenants, tant face à l'album qu'au court métrage, incitent à penser que cette combinaison écrit-oral est une bonne manière d'entrer dans la perception de la variation diatopique du français à ce niveau de maitrise.

\subsubsection{Un reportage de TV5 sur les foodtrucks au Cameroun}

27 L'activité dont il est question à présent concerne une autre cohorte d'étudiants. Le document est proposé dans un cours en laboratoire qui vise essentiellement à améliorer les compétences de compréhension orale des apprenants tout en apportant un contenu culturel. Le squelette du cours suit la progression du manuel Deux Mondes. Un certain nombre de documents authentiques puisés sur Internet sont ajoutés à la progression, par exemple de courtes vidéos illustrant des situations de la vie de tous les jours, des chansons françaises, ou encore des documents permettant une ouverture sur la francophonie. Parmi ces documents, nous avons sélectionné une vidéo présentant un foodtruck camerounais ${ }^{12}$. Dans cette vidéo, quatre personnes s'expriment: la journaliste qui n'a pas d'accent régional reconnaissable et trois personnes dont les accents sont plus ou moins prononcés. Le lexique mobilisé n'est pas spécifiquement camerounais.

Contrairement à la démarche adoptée dans l'activité précédente, le visionnement de la vidéo sur le Cameroun ne fait pas l'objet d'une préparation spécifique où le texte serait présenté et étudié avant l'écoute. Sont simplement fournies aux apprenants une courte introduction sur l'histoire du Cameroun, une carte permettant de localiser le pays, ainsi qu'une liste d'une dizaine de mots de vocabulaire en guise d'aide préalable à la compréhension. Les apprenants disposent de la fiche de niveau B1 conçue par le Cavilam $^{13}$ (voir annexe 2). Celle-ci propose le repérage de mots clés entendus dans le reportage et des activités visant à évaluer la compréhension des informations relatives aux habitudes des travailleurs camerounais, etc. Elle ne propose pas de repérer des traits linguistiques de la variation. À l'inverse, la fiche destinée au niveau A2 (annexe 1) propose une activité (activité 3) portant spécifiquement sur les énoncés produits par les trois personnes interviewées. Il s'agit d'apparier sept énoncés avec le nom du locuteur qui les prononce, ce qui implique une attention aux particularités linguistiques du Cameroun. Cette activité nous semble constituer un bon point de départ pour introduire la variation.

Les apprenants australiens ont apprécié ce document audiovisuel. Ils se sont montrés curieux et souhaitaient en savoir davantage sur la francophonie africaine. Cette 
marque d'intérêt pour la francophonie africaine concorde avec les résultats d'une recherche récente conduite auprès des enseignants des départements de français des universités australiennes (Maizonniaux et coll., 2018). Des recherches seraient nécessaires pour déterminer si ce type de document vidéo permet à long terme une sensibilisation effective à la variation diatopique, et s'il peut contribuer, associé à d'autres supports et d'autres activités, à développer les compétences de compréhension et à transformer l'image du français.

\subsection{Sensibilisation à la variation lexicale par les albums jeunesse (Suisse)}

La démarche s'adresse également à des apprenants de niveau A2-B1 et s'effectue dans le cadre de l'étude d'un album suisse destiné à la jeunesse. Sélectionné pour son contenu culturel typique, l'album Reine de Jacqueline Delaunay présente une tradition du Valais suisse, le combat de vaches, dit combat de reines. L'approche interculturelle consiste ici à comprendre l'importance de cet animal et de la tradition qui lui est liée dans les Alpes suisses. En contexte australien, les bovins occupent une place toute différente et peuvent être regroupés dans d'immenses troupeaux. Le lexique valaisan est mis en relief dans cet album grâce à un glossaire qui, tout comme l'image dans cet album de grand format, constituent des éléments facilitateurs d'importance. Dans la démarche proposée, le lexique spécifique a été présenté aux apprenants comme un élément concourant à la portée culturelle de l'album. Il n'est pas question ici de le mémoriser, mais de le reconnaitre comme faisant partie du français et de le considérer comme indexant un élément culturel d'un espace francophone. En outre, on invite les apprenants à faire usage de périphrases pour expliquer ces mots ou expressions spécifiques.

31 Comme pour le Chandail de hockey, les apprenants ont particulièrement apprécié la portée culturelle de Reine. Ils ont déclaré avoir apprécié le fait qu'il pose la question de l'identité et qu'il permet de sortir des stéréotypes liés à la Suisse. Là encore, il serait pertinent de conduire de plus amples recherches pour évaluer les effets à long terme de la sensibilisation à la variété lexicale en francophonie.

\section{Pistes de réflexion}

La démarche didactique présentée a inclus, de manière innovante, des albums et des documents filmés pour la jeunesse, généralement peu utilisés en classe de langue universitaire pour sensibiliser aux variétés du français. Sur le plan géographique, nous avons également intégré le Cameroun, pays d'Afrique francophone. La proximité de l'Australie et de la Nouvelle-Calédonie invite également à considérer la variation dans cette zone géographique : échanges lexicaux suite à la présence massive d'anglophones en Nouvelle Calédonie lors de la Seconde Guerre mondiale (Pauleau, 2016), liens entre le français de Nouvelle-Calédonie et celui de Picardie ou de Bretagne (Bottineau, 2016), résidus du français colonial du XVII ${ }^{\mathrm{e}}$ siècle, communs au français de Nouvelle-Calédonie et aux français d'Amérique (Thibault, 2016).

Alors que les manuels de langue font une place très mince à la variation et n'abordent la francophonie que sous l'angle culturel, il est possible de sensibiliser à la variation dès les niveaux A2-B1. Notre démarche a montré qu'il est possible, à partir d'un album pour 
la jeunesse et du court métrage tiré de cet album, d'aborder plusieurs aspects de la variation: la variation lexicale, morphologique et morphosyntaxique, la variation diatopique. On peut également choisir de se concentrer sur un aspect spécifique de la variation sans nécessairement aborder les autres, c'est ce que nous avons fait à partir de l'album Reine en nous penchant sur le lexique de l'album à partir du glossaire qu'il propose. Dans la perspective d'une centration sur la variation diatopique, il pourra être utile, ainsi que le suggère Falkert (2019), de partir des difficultés de compréhension que peuvent rencontrer les apprenants au lieu de proposer une approche descriptive et d'enseigner qu'un phénomène phonétique existe dans une ou plusieurs variétés. En effet, l'objectif principal de toute approche de la variation diatopique est d'améliorer la capacité des apprenants à comprendre des locuteurs francophones. Il apparait nécessaire, enfin, de développer des approches ciblées et mettre en place des dispositifs de recherche pour évaluer l'impact de démarches proposées en A2-B1 sur le court, moyen et long terme.

\section{BIBLIOGRAPHIE}

Australian CURRICUlum, ASSESSMENT AND REPORTING AUTHORITY (ACARA). (2014). Australian

Curriculum: Languages. French (revised). Disponible en ligne sur <https://

acaraweb.blob.core.windows.net/resources/F-10_Austral[...].pdf> (consulté le 29 septembre 2019).

Amireault, Valérie, Silva, Haydée, Lacelle, Nathalie, Trottet, Soline, Gladu, Ève, Allard, Renaud \& ST-PIERRE, René. (n. d.). Théâtralisation de contes et légendes du Québec - FLE/S. Disponible en ligne sur <http://litmedmod.ca/theatralisation-de-contes-et-legendes-du-quebec-fles> (consulté le 29 septembre 2019 ).

AUZEAU, Fanny. (2016). Quelles approches utiliser pour enseigner les accents francophones ? Dans C. Fréchet (dir.), La variation du français dans le monde. Quelle place dans l'enseignement? (p. 105-112). Limoges : Lambert-Lucas.

BotTinEAU, Didier. (2016). Les particularités du français calédonien (lexique, morphosyntaxe) et leurs enjeux sémantiques, pragmatiques et cognitifs. Langages, 203, 49-69.

CARRIER, Roch \& COHEN, Sheldon. (1985 [1979]). Le chandail de hockey. Montréal : Livres Toundra.

CENTRE EUROPÉEN POUR LES LANGUES VIVANTES. (n. d.). Un cadre de référence pour les approches plurielles des langues et des cultures (CARAP). Disponible en ligne sur <http://carap.ecml.at/Accueil/tabid/ 3577/language/fr-FR/Default.aspx> (consulté le 25 juillet 2017). Et également : Contes et légendes du monde, dans CARAP Kit pédagogique "Tous différents, tous égaux» (p. 91-100). Disponible en ligne sur <www.yumpu.com/fr/document/view/19632240/contes-et-legendes-du-monde-carap> (consulté le 29 septembre 2019).

COHEN, Sheldon. (1980). Le chandail de hockey. Canada : Office national du film du Canada. Disponible en ligne sur <www.youtube.com/watch?v=shyFWU8pCjs> (consulté le 29 septembre 2019). 
CONSEIL DE L'EUROPE. (2001). Cadre européen commun de référence pour les langues : apprendre, enseigner, évaluer (CECR). Paris : Didier.

DE PIETRO, Jean-François. (2004). Enjeux, orientations et perspectives pour des activités réflexives à l'école : le cas de la Suisse francophone. Dans C. Vargas (dir.), Langue et études de la langue. Approches linguistiques et didactiques (p. 81-91). Publications de l’Université de Provence.

De Pietro, Jean-François, Diemoz, Federica \& MATthey, Marinette. (2016). La variation du/des français en Suisse romande : enjeux et défis de sa présence à l'école. Dans C. Fréchet (dir.), La variation du français dans le monde. Quelle place dans l'enseignement? (p. 43-63). Limoges : LambertLucas.

DETEY, Sylvain, DURAND, Jacques, LAKS, Bernard \& LYCHE, Chantal. (2010). Les variétés du français parlé dans l'espace francophone. Ressources pour l'enseignement. Paris : Ophrys.

DETEY, Sylvain \& RACINE, Isabelle. (2012). Les apprenants de français face aux normes de prononciation : quelle(s) entrée(s) pour quelle(s) sortie(s) ? Revue française de linguistique appliquée, 17(1), 81-96.

FALKERT, Anika. (2016a). The Relevance of Accent in L2 Pronunciation Instruction: A Matter of Teaching Cultures or Language Ideologies. International Journal of Pedagogies and Learning, 11, 259-270.

FALKERT, Anika. (2016b). Accents régionaux francophones et enseignement de l'oral : quels enjeux pour la didactique du FLE ? Dans H. Kertaoui \& M. Martah (dir.), Apprendre/Enseigner le français en contextes plurilingues : des réalités sociolinguistiques aux pratiques éducatives. Actes du colloque international de Marrakech, 22-25 octobre 2014 (p. 159-178). Marrakech : Al Watanya.

FALKERT, Anika. (2019). La place de la variation dans l'enseignement de la phonétique en FLE. Réalités et perspectives. Recherches en didactique des langues et des cultures. Les Cahiers de l'Acedle, 16(1). Disponible en ligne sur <https://journals.openedition.org/rdlc/4309> (consulté le 29 septembre 2019).

FRÉCHET, Claudine. (2016). Introduction. Dans C. Fréchet (dir.), La variation du français dans le monde. Quelle place dans l'enseignement? (p. 13-15). Limoges : Lambert-Lucas.

GADET, Françoise. (2017). Les parlers jeunes dans l'île-de-France multiculturelle. Paris : Ophrys.

GALAZZI, Enrica. (2017). Variations phonétiques contemporaines : transparence et opacité dans le marché des langues globalisé. Du côté du FLE. Dans H. Tyne, M. Bilger, P. Cappeau \& E. Guerin (dir.), La variation en question(s). Hommage à Françoise Gadet (p. 291-300). Bruxelles : Peter Lang. GALAZZI, Enrica \& PATERNOSTRO, Roberto. (2016). Locuteurs de FLE en contact : un défi pour la didactique de la parole aujourd'hui. Le français dans le monde. Recherches \& applications, 60, 27-34. GRIMOLDI, Elena. (2014). Les représentations de la variation linguistique dans quelques manuels de FLE (Mémoire de master non publié). Université d'Avignon.

JEANMAIRE, Guillaume. (2017). Intégrer les variétés de français de l'espace francophone dans l'enseignement-apprentissage du FLE. The French Review, 91, 156-172.

MAINGAIN, Isabelle. (2006). Le français d'ici et d'ailleurs. Florence : Alinea Editrice.

MaIzonNiauX, Christèle. (2011). La littérature de jeunesse comme médiateur interculturel en classe de FLE. Dans J. Meunier, B. Atherton, F. Grauby \& M. Royer (dir.), Le français et la diversité francophone en Asie-Pacifique. $2^{e}$ Congrès de la Commission Asie-Pacifique-FIPF, Sydney 2010 (p. 141-150). Paris : FIPF. 
MAIZONNIAUX, Christèle. (2013). Les apports de la littérature de jeunesse pour la didactique du FLE en contexte universitaire australien (Thèse de doctorat non publiée). The Australian National University (Canberra) \& Université Stendhal-Grenoble 3, Grenoble.

MAizonniaux, Christèle, Diallo, Ibrahima, ARNEBERG-JonCAS, Justine \& MAHER, Katie. (2018). The Teaching of Francophone Africa within French Studies Programs in Australian Universities. Adélaïde : African Studies Association of Australasia and the Pacific (AFSAAP).

PATERnOSTRO, Roberto. (2016). Diversité des accents et enseignement du français. Les parlers des jeunes en région parisienne. Paris : L'Harmattan.

Pauleau, Christine. (2016). La description du français calédonien : état des lieux. Langages, 203, 21-36.

Perregaux, Christiane, De Pietro, Jean-François, De Goumoëns, Claire \& Jeannot, Dominique (dir.). (2003). ÉOLE : éducation et ouverture aux langues à l'école. Neuchâtel : CIIP.

Pillot-Loiseau, Claire. (2014). Geste vocal, voix et enseignement des langues. Dans M. Tellier \& L. Cadet (dir.), Le corps et la voix de l'enseignant : une mise en contexte théorique et pratique (p. 51-66). Paris : Maison des langues.

SHEEREN, Hugues. (2012). Variations et diversités francophones dans l'enseignement de la langue française : point de vue d'un francophone de Belgique (ou comment « défranciser » le cours de langue française). Repères-DoRiF, 1 : Le français dans le contexte plurilingue des Centres linguistiques universitaires italiens, DoRiF Università, Rome juillet 2012. Disponible en ligne sur <www.dorif.it/ ezine/ezine_printarticle.php?dorif_ezine $=5$ ef6842b02460a4c5ce5a75104166ee9\&art_id=21> (consulté le 27 septembre 2019).

SHEEREN, Hugues. (2016a). Entre norme et variation : la position inconfortable des professeurs de français langue étrangère natifs non français. Synergies France, 10, 69-81.

SHEEREN, Hugues. (2016b). Le rapport des allophones à la norme et à la variation diatopique en classe de FLE : le cas du public italien. Dans C. Fréchet. (dir.), La variation du français dans le monde. Quelle place dans l'enseignement? (p. 75-88). Limoges : Lambert-Lucas.

Terrell, Tracy D., Rogers, Mary B., KerR, Betsy J. \& Spielmann, Guy. (2013). Deux Mondes. New York : McGraw Hill.

THIBAULT, André. (2016). Diastratismes et réallocation des variantes : français d'Amérique et de Nouvelle-Calédonie. Langages, 203, 71-86.

TROTTET, Soline \& AMIREAULT, Valérie. (2013). Les contes et légendes : des discours ni tout à fait oraux, ni tout à fait écrits. Synergies Mexique, 3, 61-77.

Trottet, Soline, Amireault, Valérie, Silva, Haydée, Lacelle, Nathalie, Gladu, Ève \& Allard, Renaud. (2015). La Chasse-galerie. Parcours pédagogique. Disponible en ligne sur <http://litmedmod.ca/sites/ default/files/tclq_parcours-la-chasse-galerie.pdf> (consulté le 28 septembre 2019).

TyNe, Henry, BILGER, Mireille, CAPPEAU, Paul \& GueRIN, Emmanuelle. (2017). La variation en question(s). Hommage à Françoise Gadet. Bruxelles : Peter Lang.

VALDMAN, Albert. (1989). Classroom Foreign Language Learning and Language Variation: The Notion of Pedagogical Norms. Dans M. Eisenstein (dir.), The Dynamic Interlanguage: Empirical Studies in Language Variation (p. 261-277). New York : Plenum.

VALDMAN, Albert. (2000). Comment gérer la variation dans l'enseignement du français langue étrangère aux États-Unis. The French Review, 73(4), 648-666. 
YouTUBE. (2013). Fast-food au Cameroun. Disponible en ligne sur <www.youtube.com/watch? v=szFIQM3BufU> (consulté le 29 septembre 2019).

\section{ANNEXES}

ANNEXE 1. - Fiche proposée par le Cavilam pour le niveau A2 (vidéo : Fast-food au Cameroun)

\section{7jours}

\section{NIVEAU ÉLÉMENTAIRE / A2}

Activité 1. - Écoutez le reportage. Retrouvez l'ordre des informations données dans le reportage.

\begin{tabular}{|c|c|}
\hline $\mathrm{N}^{\mathrm{o}} \ldots . .$. & Les clients aiment manger vite et bien. \\
\hline $\mathrm{N}^{\mathrm{o}} \ldots . .$. & $\begin{array}{l}\text { Les Camerounais peuvent manger des sandwichs à base de shawarma, de légumes et de } \\
\text { sauce. }\end{array}$ \\
\hline $\mathrm{N}^{\mathrm{o}} \ldots . .$. & Au Cameroun, il y a aussi des restaurants de fast-food où on peut manger assis. \\
\hline $\mathrm{N}^{\mathrm{o}} \ldots . .$. & Les camionnettes de fast-food sont bien équipées pour faire à manger et servir les clients. \\
\hline $\mathrm{N}^{\circ} \ldots . .$. & Au Cameroun, il y a aussi des fast-foods mobiles. \\
\hline $\mathrm{N}^{\mathrm{o}} \ldots . .$. & Le secteur du fast-food est en expansion. \\
\hline $\mathrm{N}^{0} \ldots . .$. & Le phénomène de restauration rapide n'est pas nouveau au Cameroun. \\
\hline $\mathrm{N}^{0} \ldots . .$. & Quand on mange assis, on peut choisir les ingrédients du sandwich. \\
\hline
\end{tabular}

Activité 2. - Écoutez le reportage. Soulignez l'élément vu ou entendu.

\begin{tabular}{|l|l|}
\hline 1. & Une camionnette jaune est garée sur un parking / dans la rue. \\
\hline 2. & Un homme mange un sandwich, debout devant un magasin / une camionnette. \\
\hline 3. & Deux cuisiniers préparent des sandwichs / des hamburgers. \\
\hline 4. & Un cuisinier coupe de la viande / du poisson et des tomates. \\
\hline 5. & Un homme montre à la caméra qu'il est content de son sandwich / déçu par son sandwich. \\
\hline 6. & Un sandwich coute en moyenne $1,50 €$ / $2,50 €$. \\
\hline 7. & Il y a peu / il y a beaucoup de Camerounais qui choisissent ce mode de restauration. \\
\hline
\end{tabular}


Activité 3. - Écoutez le reportage. Retrouvez les propos de chaque personne interviewée.

\begin{tabular}{|l|l|l|l|}
\hline & & $\square$ & Les travailleurs ont besoin de manger rapidement. \\
\hline Adèle Koundji & $\square$ & $\square$ & Je peux voir ce que je vais manger. \\
\hline & & $\square$ & Les gens adorent manger de nouvelles choses. \\
\hline Jacques Moukoko & $\square$ & $\square$ & C'est moins cher que le restaurant. \\
\hline & & $\square$ & Les travailleurs habitent loin de leur domicile. \\
\hline Serge Ngajui Fosso & $\square$ & $\square$ & $\begin{array}{l}\text { C'est la première fois que les Camerounais voient un kebab dans une } \\
\text { voiture. }\end{array}$ \\
\hline & & $\square$ & La camionnette de fast-food, ça marche bien. \\
\hline
\end{tabular}

Activité 4. - En vous aidant du reportage, retrouvez le contraire de chaque adjectif.

1. Le reportage parle du phénomène de restauration lente $\neq$

2. Au Cameroun, ce phénomène n'est pas vieux $\neq$

3. On trouve maintenant des fast-foods fixes $\neq$

4. Certains Camerounais préfèrent encore manger debout $\neq$

5. Les citadins ont de vieilles habitudes alimentaires $\neq$

Activité 5. - Présentez un plat bon marché de votre pays que l'on peut manger rapidement.

ANNEXE 2. - Fiche proposée par le Cavilam pour le niveau B1 (vidéo : Fast-food au Cameroun)

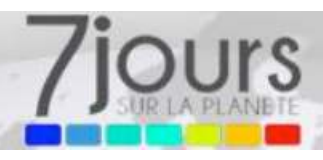

\section{NIVEAU INTERMÉDIAIRE / B1}

Activité 1. - Écoutez le reportage et cochez les mots-clés que vous entendez.

\begin{tabular}{|l|l|l|l|l|l|l|l|l|l|}
\hline$\square$ & Le phénomène & $\square$ & L'alimentation & $\square$ & La restauration & $\square$ & Des sandwichs & $\square$ & Des hot-dogs \\
\hline$\square$ & Une camionnette & $\square$ & Le kebab & $\square$ & Les Congolais & $\square$ & Les Camerounais & $\square$ & Le menu \\
\hline$\square$ & La formule & $\square$ & Les ingrédients & $\square$ & Les légumes & $\square$ & Ancien & $\square$ & Ordinaire \\
\hline$\square$ & En augmentation & $\square$ & La place & $\square$ & L'attitude & $\square$ & Les habitudes & $\square$ & En expansion \\
\hline
\end{tabular}


Activité 2. - Écoutez le reportage et dites si ces affirmations sont vraies ou fausses.

\begin{tabular}{|l|l|l|l|}
\hline \multicolumn{2}{|l|}{} & Vrai & Faux \\
\hline 1. & La restauration rapide est un phénomène nouveau au Cameroun. & & \\
\hline 2. & Le sandwich coute moins de deux euros. & & \\
\hline 3. & $\begin{array}{l}\text { Manger son sandwich à base de shawarma debout dans la rue n'est pas une pratique } \\
\text { nouvelle pour les Camerounais. }\end{array}$ & & \\
\hline 4. & $\begin{array}{l}\text { Le propriétaire du restaurant a eu l'idée de la camionnette grâce à un séjour de } \\
\text { quatre ans au Mali. }\end{array}$ & & \\
\hline 5. & $\begin{array}{l}\text { Les Camerounais peuvent aussi manger leur sandwich assis, dans un restaurant. } \\
\text { 6. }\end{array}$ & Les restaurateurs mettent en avant la possibilité d'ajouter des légumes frais dans \\
les sandwichs. & & \\
\hline 7. & $\begin{array}{l}\text { En général, les travailleurs n'ont pas beaucoup de temps pour déjeuner à midi. } \\
\text { 8. }\end{array}$ & & \\
\hline villes. & & \\
\hline
\end{tabular}

Activité 3. - En vous aidant du reportage, retrouvez les avantages de la camionnette fast-food.

C'est bon marché. C'est meilleur. On peut marcher en même temps. C'est plus convivial. C'est plus rapide.

C'est bien quand je suis pressé. On sait ce qu'on mange. C'est un peu plus cher.

Activité 4. - Écoutez le reportage. Complétez le résumé proposé.

Depuis plusieurs années, rapide attire de plus en plus les

Camerounais. Aujourd'hui, on peut même trouver mobiles qui proposent identiques aux restaurants fixes. Les Camerounais aiment les nouvelles choses et tout ce qui sort de de fast-foods mobiles se développe de plus de plus en plus. Le secteur est

Activité 5. - Une camionnette de vente ambulante vient de s'installer au coin de votre rue. Il est $19 \mathrm{~h}$, vous avez faim. Imaginez le dialogue avec le cuisinier / la cuisinière pour savoir ce qu'il propose à manger. 


\section{NOTES}

1. Pour plus de détails, se référer à : < www.projet-pfc.net/le-projet-pfc-ef/ressourcesdidactiques/fichespedago/> (consulté le 29 mai 2019)

2. «À partir du niveau B2, les apprenants sont capables de s'exprimer de manière adéquate dans une langue appropriée aux situations et aux acteurs sociaux et ils commencent à acquérir la capacité de faire face aux variations du discours et de mieux maitriser le registre et l'expression. » (Conseil de l'Europe, 2001, p. 95)

3. Se référer à «Foundation to Year 10 sequence » («Programmes allant de la grande section de maternelle à la seconde ", notre traduction).

4. Correspondance personnelle.

5. «Understanding-Analysing and understanding language and culture as resources for interpreting and shaping meaning in intercultural exchange ».

6. Sheeren parle également $d u$ «monocentrisme déroutant» des enseignants de français (pour plus de détails, se référer à Sheeren, 2016 b).

7. Pour une description plus complète de ces ressources, se référer à Jeanmaire (2017).

8. Les sombres légendes de la terre - Le diable de la chasse-galerie, de Félix Roque, diffusé sur TV5 Canada. Disponible en ligne sur <https://www.youtube.com/watch?v=OuHkRWO1obM> (consulté le 29 mai 2019).

9. Cette sensibilisation à l'accent camerounais a été conduite à Adélaïde (Flinders University) dans le cadre d'un cours de laboratoire. Pour ce qui est du travail effectué à partir des albums pour la jeunesse, il a été effectué à Canberra (The Australian National University). Ce travail faisait partie d'une recherche doctorale sur les apports de la littérature de jeunesse pour l'enseignement du FLE.

10. La question de la portée culturelle de cet album a été abordée dans Maizonniaux (2011).

11. Les exercices préparatoires proposés en amont du visionnement du court métrage ont porté sur la compréhension (compréhension de la trame narrative de l'album, des expressions spécifiquement québécoises, des spécificités culturelles et du contexte socio-historique). Ils ont également porté sur l'expression (exercices de type «rappel», exercices portant sur les informations transmises par l'image et/ou le texte, ou sur les diverses interprétations possibles de l'album). Une attention particulière a été accordée, au niveau culturel, à la mise en exergue de l'implicite (les relations historiques et rivalités entre anglophones et francophones au Québec), et à la langue française comme partie intégrante et fondamentale de l'identité québécoise. Pour plus de détails sur la démarche didactique, voir Maizonniaux (2013).

12. < www.youtube.com/watch?v=szFIQM3BufU> (consulté le 29 mai 2019). Cet extrait a été présenté sur le site de TV5 Monde, 7 jours sur la planète.

13. Le CAVILAM (Centre d'approches vivantes des langues et des médias) propose en ligne des propositions pédagogiques à destination des professeurs de français langue étrangère. Le site Enseigner le français avec TV5 Monde propose des vidéos accompagnées de fiches pour le professeur et les élèves.

\section{RÉSUMÉS}

La plupart des manuels de FLE invitent désormais à sensibiliser les apprenants à la diversité culturelle des régions francophones, et ce, à travers des pages consacrées à certains aspects de la 
culture de ces pays. Cependant, ils n'incluent pas toujours de sensibilisation à un lexique spécifique ou aux accents régionaux francophones (Falkert, 2016a). Le CECR intègre une sensibilisation à la variation à partir du niveau B2, mais certains chercheurs (Falkert, 2016b ; Auzeau, 2016) sont d'avis que celle-ci devrait apparaitre à des niveaux moins élevés de maitrise de la langue cible. Nous questionnerons ici la pertinence d'une approche spécifique proposée à des apprenants de niveau A2-B1 basée sur des documents authentiques provenant du Québec et d'Afrique francophone.

These days most textbooks developed for the teaching of French as a foreign language aim to increase learner's awareness of the cultural diversity of French-speaking regions, primarily through pages devoted to aspects of the culture of the countries concerned. However, and as noted by Falkert (2016a), such approaches do not always promote awareness to a specific lexicon nor invite the student to listen to and understand regional French accents. The CECR includes awareness to variation at level B2 but some researchers (Falkert, 2016b; Auzeau, 2016) claim that approaches incorporating such variation should be included at lower levels of mastery of the target language. We will question the relevance of a specific approach targeting students at A2B1 level through the use of authentic documents from Quebec and francophone African countries.

INDEX

Mots-clés : variation, médiation, lexique, prononciation, niveau A2-B1 du CECR

Keywords : variation, mediation, vocabulary, pronunciation, CEFR level A2-B1

\section{AUTEUR \\ CHRISTĖLE MAIZONNIAUX}

Flinders University, Adélaïde, Australie 